\title{
EXPECTATIVA DE MULHERES À ESPERA DE REPRODUÇÃO ASSISTIDA EM HOSPITAL PÚBLICO DO DF — ESTUDO BIOÉTICO
}

\author{
Mônica Samrsla, Juliana Cezar Nunes, Carolina Kalume, Antônio Carlos Rodrigues da Cunha, Volnei Garrafa* \\ Trabalho realizado na Cátedra Unesco de Bioética, Centro de Estudos Avançados Multidisciplinares, Universidade de Brasília, Distrito Federal
}

\author{
*Correspondência: \\ Cátedra Unesco de Bioética \\ Universidade de Brasília \\ Caixa postal 04451 \\ Brasília/DF \\ Cep: $70.904-970$ \\ Tel/fax: (61) 3307-3946 \\ bioetica@unb.br
}

\begin{abstract}
RESUMO
Oвjetivo. Investigar a expectativa de mulheres que aguardam tratamento de reprodução assistida (RA) no hospital de referência da rede pública de saúde do Distrito Federal, Brasil, o Hospital Regional da Asa Sul (HRAS).

Métodos. Durante um mês, foram entrevistadas, por meio de questionário composto por dez perguntas objetivas, 51 das 56 mulheres que procuraram o HRAS em busca de tratamento para infertilidade.

Resultados. A amostra foi dividida em dois grupos. 0 primeiro, considerado grupo controle, constou de 27 pacientes recém-encaminhadas de postos de saúde ou de um hospital regional para o HRAS. O segundo, identificado como grupo de estudo, teve 24 mulheres já inscritas na fila de espera para fertilização in vitro, com diagnóstico anterior concluído pela equipe médica especializada do hospital. O longo tempo médio de espera pelo tratamento faz com que as mulheres avancem na idade e acabem ingressando no período de gravidez de risco sem terem conseguido tratamento.

Conclusão. As mulheres sem condições de arcar com despesas de tratamento em clínicas privadas de infertilidade estão longe de se beneficiar da RA; o problema de saúde dessa população espećíica não passa por um processo de correção e muito menos de distribuição de recursos. A espera imposta pelo Estado potencializa a expectativa das pacientes à espera de tratamento. Não há um padrão de informação sobre o tempo da espera. A imprevisibilidade no repasse de medicamentos indispensáveis para a fertilização in vitro coloca o oferecimento futuro do serviço em dúvida.
\end{abstract}

Unitermos: Bioética. Sistema Único de Saúde. Reprodução assistida. Eqüidade. Prioridades.

\section{INTRODUÇÃO}

O presente estudo visa avaliar a expectativa de mulheres que aguardam tratamento de reprodução assistida (RA) no hospital público de referência da rede de saúde do Distrito Federal, DF, Brasil, integrante do Sistema Único de Saúde (SUS). Muitas mulheres e homens com problemas de fertilidade ainda se deparam com condições econômicas desfavoráveis para arcar com as despesas de um tratamento que chega a custar $R \$ 12$ mil por tentativa. No DF, depois de passar pelo posto de saúde e/ou hospital regional, o casal é encaminhado ao Hospital Regional da Asa Sul (HRAS), em Brasília. De acordo com a coordenação do serviço, existiam 1865 pacientes inscritas na fila de espera para fertilização in vitro em novembro de 2004.

Instituído pela Constituição Federal de 1988', o SUS preconiza o acesso integral, universal e eqüitativo à saúde como direito de cidadania. A Declaração Universal dos Direitos Humanos (DUDH) de 1948 , no artigo XVI, prevê: "Os homens e as mulheres de maior idade, sem qualquer restrição de raça, nacionalidade ou religião, têm o direito de contrair matrimônio e fundar a família" ${ }^{2}$. Entendendo a família como marido, mulher e filhos, este estudo pretende abordar a RA a partir de um enfoque de Bioética e direitos humanos. Para isso, a rotina do ambulatório de RA - HRAS foi acompanhada durante um mês e as pacientes que concordaram em participar do estudo responderam a um questionário.

\section{Reprodução assistida - breve histórico}

Na busca pela transcendência, o ser humano costuma ver nos filhos a perpetuaçãa da própria existência. Por muitos séculos, esse desejo fazia parte do espaço privado do casal. Homens e mulheres que não conseguiam ter filhos por meio de relação sexual estavam aprisionados a essa condição. O significado da infertilidade para o casal vem sendo objeto de vários estudos. Ao longo de suas trajetórias de vida, as mulheres vão se tornando mães e transformam a maternidade em uma experiência de continuidade, natural e fundamental na noção de feminilidade ${ }^{3}$. Os homens, por sua vez, não concebem a paternidade como construção do passado. Eles relacionam a reprodução a uma situação do presente (casamento), chave para a confirmação da sexualidade e virilidade.

Cientes do que representa a restrição à liberdade de procriar, os cientistas passaram a desenvolver diferentes estudos. Lázaro Spallanzani, no final do século XVII, conseguiu fecundar uma cadela em cio após coletar o sêmen de um cachorro. O método passou a ser aplicado em 1790, quando o médico John Hunter inseminou artificialmente uma mulher utilizando o sêmen do marido. Até então, o óvulo continuava a ser fecundando dentro do corpo. Essa fronteira foi ultrapassada em 1978, na Inglaterra, quando nasceu o primeiro bebê de proveta: Louise Brown. Desde então, várias outras técnicas de fertilização in vitro foram desenvolvidas para facilitar a reprodução fora do corpo humano. No Brasil, o primeiro bebê de proveta nasceu em 
outubro de 1984. O interesse pela técnica aumenta à medida que cresce a participação da mulher no mercado de trabalho e o desejo de adiar a maternidade para depois dos 30 anos, época em que o aparelho reprodutivo já iniciou seu processo de envelhecimento. As novas tecnologias, por sua vez, potencializam as chances de um casal com problemas ter filhos.

$\mathrm{Na}$ tentativa de evitar excessos, o Conselho Federal de Medicina (CFM) publicou a Resolução I358, em 19924. O documento considera a infertilidade humana como um problema de saúde. Esta resolução, pela necessidade de harmonizar o uso das técnicas com os princípios da ética médica, preconiza a utilização da RA apenas nos casos em que outros tratamentos não são possíveis. Fixa em quatro o número máximo de embriões a serem inseminados; assegura a preservação do anonimato para os doadores de gametas ou pré-embriões e proíbe esse tipo de prática com caráter comercial. O CFM não autoriza pré-seleção de sexo ou qualquer outra característica biológica.

No Congresso Nacional, tramitam com lentidão espantosa quatro Projetos de Lei (PL) a respeito da RA $A^{5}$. Nenhum deles discute o acesso aos tratamentos na rede pública de saúde, somente abordam questões técnicas. O PL I | 84/2003 impede o congelamento de embriões, limita em dois o número de embriões implantados por tentativa, impede o uso da técnica de útero de substituição (barriga de aluguel) e torna obrigatória a identificação de doadores de óvulos e espermatozóides. Caso aprovado, esse PL vai regular uma atividade já exercida de forma significativa em clínicas particulares. Atualmente, no Brasil, existem apenas seis unidades de saúde pública oferecendo o tratamento de RA. Em 2003, das 117 clínicas cadastradas na Sociedade Brasileira de Reprodução Assistida (SBRA), nada menos que 47\% estavam concentradas no estado de São Paulo e, destas, 54\% estavam localizadas na capital| ${ }^{6}$. Calculava-se, há três anos, em 7 mil o número de crianças nascidas a partir de técnicas de fertilização no Brasil.

Um dossiê sobre RA produzido pela Rede Feminista de Saúde chama a atenção para a falta de regulação e da "privatização da medicalização da fecundidade feminina", que contribuem para o encobrimento de estudos isentos sobre "efeitos colaterais de medicações, número de embriões produzidos, implantados, descartados, congelados, proporção de gestações múltiplas e condições dessas gestações" "7.

No Distrito Federal, existem três clínicas de RA cadastradas na SBRA. Apenas uma unidade pública de saúde - o HRAS - possui laboratório capaz de oferecer fertilização in vitro. O setor de reprodução humana do hospital foi fundado há cerca de 20 anos. Porém, a RA, incluindo fertilização in vitro, só passou a ser disponibilizada em agosto de 1998. De acordo com a coordenação do serviço existiam, em novembro de 2004, 1865 pacientes inscritas na fila de espera para fertilização in vitro; o único critério de seleção é a ordem de inscrição. De acordo com informações dos funcionários do serviço, o Estado disponibiliza por mês material suficiente para executar apenas dez ciclos completos de tratamento.

\section{Direitos humanos e saúde reprodutiva}

A partir da Declaração Universal dos Direitos Humanos, a atual concepção dos direitos reprodutivos não se limita à simples proteção da reprodução, mas defende um conjunto de direitos individuais e sociais, que devem interagir em busca do pleno exercício da sexualidade e reprodução humana. Essa nova concepção tem como ponto de partida uma perspectiva de igualdade e eqüidade nas relações pessoais e sociais e a ampliação das obrigações do Estado na promoção, efetivação e implementação desses direitos ${ }^{8}$.

A saúde reprodutiva implica, por conseguinte, que o indivíduo possa ter uma vida sexual segura e satisfatória, com capacidade de reproduzir e liberdade de decidir sobre quando, como e quantas vezes deve fazê-lo. Ter o direito de informação e acesso a métodos eficientes, seguros e financeiramente compatíveis de planejamento familiar? bem como a serviços públicos apropriados que garantam as melhores condições para gestação e parto, incluindo acesso a técnicas avançadas como a RA. De acordo com o Art. XXV - 2 da DUDH, "...a maternidade e a infância têm direito a cuidados e assistência especiais". Segundo Vanderplaat, "o direito à saúde contém liberdades e prerrogativas. As liberdades incluem direito de controlar sua própria saúde e corpo, incluindo liberdade sexual e reprodutiva. Ao contrário, as prerrogativas incluem o direito a um sistema de proteção à saúde que ofereça igualdade de oportunidades para as pessoas usufruírem do mais alto nível de saúde sustentável"10.

O plano de ação da Conferência Internacional sobre População e Desenvolvimento, realizada no Cairo em 1994, e o documento resultante da IV Conferência Mundial da Mulher, que ocorreu em Pequim, em 1995, legitimaram o conceito de direitos reprodutivos na sua concepção atual'. O documento do Cairo destaca como direitos humanos básicos: decidir livre e responsavelmente sobre o número, o espaçamento e a oportunidade de ter filhos; ter acesso à informação e aos meios para decidir; e gozar do mais elevado padrão de saúde sexual e reprodutiva.

\section{Reprodução assistida no Brasil}

A história brasileira foi marcada por uma cultura religiosa cristãcatólica, que transpôs para o plano normativo a obediência e a servidão da mulher em relação ao homem e à procriação de tantos filhos quantos Deus e a natureza determinassem. Na década de 1970, a idéia da existência de uma sub-raça brasileira, decorrente da pobreza e de famílias numerosas e de seus malefícios para a segurança nacional e desenvolvimento, suscitou projetos não oficiais, mas consentidos pelo poder autoritário, que estimularam esterilizações em massa no País. É nesse contexto que surge a Sociedade Civil de Bem-Estar Familiar (Bemfam) no Brasil, entidade privada que desenvolvia, com o apoio do governo militar, projetos para difundir as vantagens de uma família reduzida e o uso de práticas anticonceptivas modernas".

Tal política restritiva passou a ser questionada nos anos 80.0 movimento feminista começou a denunciar casos de esterilizações injustificadas e precoces. Em 1983, enquanto o Congresso Nacional instaurava uma Comissão Parlamentar de Inquérito (CPI) para investigar as denúncias, o Poder Executivo criou o Programa de Assistência Integral à Saúde da Mulher (Paism). Por meio dele, assumiu a assistência social e integral à saúde da mulher, principalmente em questões referentes ao planejamento familiar e à sexualidade.

As esterilizações voltaram a ser debatidas em meados dos anos 90 , quando uma nova CPI foi criada para apurar denúncias de excesso. Não regulamentadas, as esterilizações cirúrgicas acabavam sendo feitas de forma abusiva e descuidada. Os trabalhos da CPI resultaram em um 
Projeto de Lei, aprovado pelo Congresso e publicado no Diário Oficial da União, em 1997. Desde então, casais interessados em se submeter a procedimentos de esterilização devem passar por um processo de até dois meses, que inclui entrevista, palestras e aconselhamento. No entanto, a cultura da esterilização, estabelecida nas décadas anteriores, ainda mostra seu impacto. Dados do Ministério da Saúde revelam que, entre 1999 e 2004, o número de cirurgias de laqueadura e vasectomia aumentou 20 vezes. Por outro lado, várias pesquisas em curso apontam para a existência de casais esterilizados no passado e que hoje buscam nos hospitais públicos ajuda para engravidar ${ }^{2}$. $\mathrm{O}$ índice de arrependimento, de acordo com dados da Universidade de Campinas, é 12\% entre as mulheres que fazem laqueadura ${ }^{13}$.

De alguma forma, a demanda por serviço de reprodução assistida foi detectada pelo Ministério da Saúde que, em fevereiro de 2005, anunciou a intenção de ampliar as ações na área de planejamento familiar, priorizando métodos anticoncepcionais reversíveis e regularizando a oferta de reprodução assistida. As novas ações atenderiam 60 milhões de mulheres em idade fértil. De acordo com a imprensa, pelos cálculos do Ministério, os técnicos trabalham com um número de 7 milhões de pessoas inférteis no Brasil ${ }^{14}$.

\section{Métodos}

O Hospital Regional da Asa Sul, de Brasilia, DF, foi tomado como referência para o presente estudo. O instrumento para a coleta dos dados foi um questionário composto por dez perguntas objetivas relacionadas ao assunto. A pesquisa foi aprovada pelo Comitê de Ética em Pesquisa da Faculdade de Ciências da Saúde da Universidade de Brasília, incluindo o termo de consentimento livre e esclarecido, que foi assinado por todas as mulheres pesquisadas.

Durante 30 dias, todas as 56 mulheres que procuraram o HRAS em busca de tratamento para infertilidade foram convidadas a participar da pesquisa. Cinco pacientes não concordaram em participar do estudo. Os pesquisadores dividiram a amostra restante em dois grupos. 0 primeiro, considerado como grupo de controle, foi formado por 27 pacientes recém-encaminhadas de postos de saúde ou de um dos hospitais regionais do DF para o hospital público de referência. Para sua inclusão no programa da RA, restam a elas ainda alguns exames comprobatórios das causas de infertilidade do casal. O segundo grupo preenche o pré-requisito estabelecido pelos objetivos deste estudo, ou seja, mulheres já inscritas na fila de espera para fertilização in vitro, com diagnóstico concluído. Fazem parte do grupo de estudo 24 mulheres. Essas mulheres freqüentam habitualmente o hospital para se submeterem a exames ginecológicos de rotina. O total de sujeitos participantes da amostra, portanto, foi de 51 .

Para os dois grupos, foram entregues questionários com dez perguntas a serem respondidas de forma objetiva com um " $x$ " no espaço que corresponde à realidade individual. As perguntas foram: Qual é sua idade? Quantos filhos você tem atualmente? Qual sua renda familiar mensal? Quando você procurou ajuda para engravidar nessa unidade de saúde, foi informada sobre o tempo de espera para conseguir o tratamento? Se a resposta anterior foi sim, qual foi a média de tempo informada pelos profissionais que the atenderam? Há quanto tempo espera por um tratamento para engravidar com ajuda médica?
Você sabe quantas pessoas aproximadamente aguardam o mesmo tratamento? Você acha que o sistema público tem obrigação de oferecer tratamento para engravidar? Você tem esperança de ser chamada para se submeter ao tratamento para engravidar nessa unidade de saúde? Você doaria seus óvulos em troca de tratamento para engravidar?

\section{Resultados}

\section{Grupo I - controle}

Composto por mulheres em fase de investigação das causas de infertilidade do casal, a maior parte delas (63\%) pertencente à faixa etária que compreende as idades de 26 a 35 anos. Das 27 pacientes deste grupo, 17 não possuíam filhos e 7 possuíam apenas um filho. As opções dois, três ou acima de três filhos receberam uma resposta cada. Em relação à renda, 37\% das mulheres desse grupo têm renda familiar mensal entre $R \$ 260$ e $R \$ 520$ e 44\% entre $R \$ 520$ e $R \$ 780$. Ou seja, as pacientes do grupo controle têm renda familiar variando entre um e três salários mínimos. Sobre o tempo de espera pelo tratamento, as respostas foram equânimes: 13 mulheres disseram que sim, foram informadas sobre o tempo de espera para engravidar nesta unidade, e as outras 14 disseram que não. Entre as 13 que receberam informação, o tempo de espera se concentrou preferencialmente na faixa de um a dois anos (quatro mulheres) e de dois a quatro anos (quatro mulheres).

Como o grupo controle reúne mulheres em fase inicial de acompanhamento no HRAS, o estudo constatou que 59\% das pacientes aguardavam havia menos de seis meses para iniciar a bateria final de exames, com vistas a confirmar as causas de infertilidade do casal e iniciar a espera pelo tratamento propriamente dito. Verificou-se que apenas uma das 27 mulheres do grupo controle sabia quantas pessoas aguardavam o mesmo tratamento, e apenas quatro não achavam que o sistema público de saúde teria obrigação de oferecer RA. Todas as mulheres desse grupo tinham esperança de serem chamadas para se submeter a uma intervenção que pusesse fim à infertilidade do casal. Ao serem questionadas sobre a possibilidade de doar os óvulos excedentes em troca de financiamento para engravidar, as mulheres do grupo dividiram-se de forma equânime: I 4 estariam dispostas a doar e I 3 não.

\section{Grupo 2 - estudo}

Composto por 24 pacientes em fase de espera por tratamento, com diagnóstico já concluído. As faixas etárias predominantes foram de 26 a 35 anos (37,5\%) e de 36 a 45 anos (37,5\%). Foi constatado também que a maior parte das mulheres desse grupo $(79,1 \%)$ não possuía filhos. A renda familiar dessas pacientes estava concentrada entre $R \$ 260$ e $R \$ 520$ (45,8\%). Das mulheres que participaram do estudo, 62,5\% não foram informadas na unidade pública de referência sobre o tempo médio de espera pelo tratamento. Das nove que foram informadas, cinco receberam a informação de que o tempo médio de espera variava de dois a quatro anos. Apenas uma disse que foi informada que esse tempo poderia ser superior a quatro anos.

Ao serem perguntadas sobre o tempo já transcorrido de espera, $30 \%$ das entrevistadas disseram que esperavam entre dois e quatro anos e $30 \%$ afirmaram que esperavam havia mais de quatro anos. A maior parte das pacientes (75\%) não sabia quantas pessoas aguardavam 
o mesmo tratamento naquela unidade de saúde. Três das 24 entrevistadas não acreditavam que o sistema público de saúde teria obrigação de lhes oferecer RA. Nesse grupo, apenas uma mulher afirmou não ter esperança de ser chamada para engravidar na unidade.

\section{Discussão}

A divisão da amostra em dois grupos permitiu observar as semeIhanças e diferenças de expectativas e vivências entre as mulheres que ainda não entraram na lista de espera por fertilização in vitro e as que já estão na fila. Observou-se que, nos dois grupos, a maior parte das mulheres não possui filhos e tem renda familiar entre um e três salários mínimos. A faixa etária predominante no grupo controle é mais baixa do que a do grupo de estudo; enquanto $63 \%$ das mulheres do grupo 1 estavam na faixa entre 26 e 35 anos, no grupo 2 esse índice cai para $37,5 \%$. A busca pelo tratamento se dá no período fértil da mulher, quando o corpo oferece melhores condições para a reprodução. O tempo de espera pelo tratamento, no entanto, faz com que as mulheres avancem nas faixas etárias e corram o risco de ingressar no período de gravidez de risco sem terem conseguido o tratamento.

Filósofos da Antigüidade já diferenciavam justiça corretiva e distributiva, preocupados com o tratamento igualitário entre as pessoas. Os resultados da pesquisa demonstram que as mulheres sem condições de arcar com as despesas de tratamento em clínicas de infertilidade estão cada vez mais longe de se beneficiar dos ideais de justiça. O problema de saúde dessa população não passa por um processo de correção ou de distribuiçãa de recursos. Pelo contrário, com o correr do tempo, os limites físicos impedem que o Estado, após impor anos de espera, desempenhe um papel corretivo ou distributivo.

Os dados do estudo mostram, inclusive, que essa espera imposta pelo Estado potencializa ainda mais a expectativa das pacientes em relação à RA. Não há sequer um padrão para informá-las sobre o tempo da espera, uma vez que a imprevisibilidade no repasse de medicamentos para a fertilização in vitro coloca até mesmo o oferecimento do serviço em dúvida. Em 2002, a unidade passou seis meses sem receber suprimento $^{15}$. Nos dois grupos identificados, em média, o tempo informado de espera pelo tratamento foi de dois a quatro anos. No entanto, o grupo de estudo relatou que o tempo de espera médio real não coincide com o tempo informado. Quando entrevistadas, 30\% das mulheres já inscritas na fila informaram que estão havia mais de quatro anos aguardando tratamento.

Harris, citado por Garrafa ${ }^{16}$, diz que quando os recursos são escassos, é possível proceder mudanças particulares nos níveis de escassez, seja por meio de estratégias que permitam fazer frente à mesma ou por aumento nas cotas orçamentárias destinadas à assistência sanitária. E ainda: "...uma prioridade importante é aquela de individualizar soluções morais com as quais se possa enfrentar a escassez, soluções que não comportam nem a discriminação injusta, nem a tirania da maioria".

O sistema social é um sistema de cooperações que visa obter vantagens mútuas, e o Estado, o responsável pela distribuição dos bens sociais ${ }^{17}$. Segundo Rawls, citado por Lacoste ${ }^{18}$, eqüidade equivale a retidão ou sentido de justiça natural, cujo objetivo, no âmbito sanitário, é a provisão igualitária de serviços para toda a população: "....não existe injustiça no fato de um pequeno número obter vantagens superiores à média, contanto que justamente por aí... a situação dos mais desfavorecidos seja melhorada".

Para Sen ${ }^{19}$, a eqüidade deve ser entendida como igualdade de oportunidades; para sua efetiva consecução, torna-se necessário compensar as desigualdades de capacidades. Eqüidade, no contexto do Estado, é oferecer os recursos de saúde de acordo com a necessidade de cada um, incluindo a garantia de acesso aos serviços de média e alta complexidade. Em outras palavras: "A eqüidade, ou seja, o reconhecimento de necessidades diferentes, de sujeitos também diferentes, para atingir direitos iguais, é o caminho da ética prática em face da realização dos direitos humanos universais... representado neste debate pela possibilidade de acesso à saúde" 20 .

Nos dois grupos analisados, a maior parte das pacientes considera um dever do Estado assegurar o direito à reprodução e têm esperança de conseguir o tratamento. Por outro lado, tanto as mulheres do grupo controle quanto as do grupo de estudo não sabem quantas pessoas vivem a mesma espera. A falta de consciência de grupo, associada ao longo tempo de espera na fila, pode ser considerada variável importante para o fato de a maior parte das mulheres do grupo 2 expressar vontade de doar óvulos em troca de financiamento para o próprio tratamento. Tal maioria não foi identificada no grupo controle.

De acordo com Berlinguer ${ }^{21}$, a educação e a consciência de grupo são importantes para o exercício da Bioética: uma sociedade forte é feita por indivíduos fortes: "Pensar eticamente é agir e mudar modelos para exercer o direito de controle social... uma sociedade organizada e forte pode garantir um acesso igual aos serviços de saúde e permitir a eqüidade no atendimento de suas necessidades".

A Bioética serve primordialmente como uma reflexão em torno de práticas sociais e, à medida que envolve os indivíduos, deve estimular que estes devam recuperar sua capacidade de indignação. A qualidade de vida é a própria vida e, se não houver um coletivo forte, a autonomia individual fica ameaçada: "A Justiça é o ordenamento social que permite a cada membro cobrir suas necessidades e manter suas opções de projeto de vida. (...) Para ter acesso a um eventual direito à educação, de trabalho ou livre migração, o indivíduo tem de estar sadio de forma tal que o direito à atenção médica seja o primeiro e mais fundamental que deveria estar à disposição da cidadania"22.

O Conselho Nacional de Saúde declarou que "...a construção da eqüidade na área da saúde consiste, basicamente, em investir na oferta de serviços para grupos populacionais com acesso e utilização insuficientes, entre outros mecanismos, sem reprimir a demanda de serviços necessários a segmentos e grupos que já têm o acesso garantido"23. Assim, o Estado brasileiro tem o dever de oferecer e o cidadão direito do acesso aos bens e serviços oferecidos, sem considerar as diferenças individuais. Neste sentido, à luz da Bioética, é preciso considerar o desejo de mulheres realizarem - sonho da maternidade e a demora do sistema público em corresponder a esse anseio de alto custo social.

O biomercado tecnológico e a complexidade das escolhas morais surgidas do progresso científico devem nos levar a "discutir e raciocinar sobre os casos especiais, para chegarmos a uma reflexão moralmente mais aceita, uma vez que a análise deve sempre partir da relação saúde e sociedade, para só então podermos chegar a conclusões bioéticas"2! . 
Dessa forma, devemos avaliar a saúde como uma questão social e, a partir daí, sustentar a necessidade de desenvolvimento de políticas populacionais mais justas.

Durante a aplicação dos questionários, observou-se alguns dados que merecem destaque. Todas as pacientes entrevistadas tinham parceiro fixo (marido ou companheiro) havia mais de um ano - algumas até 12 anos. É freqüente a busca por tratamento de fertilização in vitro após laqueadura, demonstrando, em grande parte dos casos, o despreparo dessas mulheres na hora de optar por não mais ter filhos e submeter-se a um procedimento radical de esterilidade ${ }^{12}$. Entre as mulheres que nunca tiveram uma gestação completa, havia significativa ocorrência de adoção ou, pelo menos, reflexão sobre o assunto.

Outro dado que chamou a atenção durante o desenvolvimento da pesquisa foi a localização dos consultórios do setor de RA do HRAS. As salas estão situadas no mesmo corredor de atendimento obstétrico, onde mulheres grávidas exibem seu estado tão desejado pelo grupo anterior. A situação de expectativa e ansiedade gerada nestas mulheres foi expressa em comentários como: "...um dia também vou ter uma barriga como esta". Além disso, no mês em que a pesquisa foi realizada, um novo ambulatório começou a funcionar, no mesmo local, voltado para crianças com problemas de respiração bucal. A organização física do ambulatório, portanto, evidencia que, neste ponto, a humanização apregoada pelos atuais gestores do SUS não está sendo colocada em prática na oferta do serviço em estudo.

\section{Conclusão}

Os avanços nas técnicas de RA trouxeram para casais inférteis novas perspectivas e, sobretudo, esperanças de realizar o desejo de procriação. Ao mesmo tempo, a falta de recursos do SUS suscita uma série de dilemas, conflitos e ansiedades. O presente estudo constatou que as pacientes não recebem respostas uniformes sobre o tempo médio de espera pelo tratamento. Tampouco sabem da existência de outras mulheres na mesma situação de espera no serviço. Apesar da baixa média de escolaridade e nível socioeconômico, as pacientes à espera de RA acreditam que serão beneficiadas pelo Estado. A pesquisa demonstra, no entanto, que apesar de buscarem o exercício de cidadania, usufruindo dos seus direitos, as pacientes tinham baixa ou quase nenhuma noção de grupo.

O isolamento dificulta a organização para mobilizar e alertar as autoridades quanto à demora no tratamento e à falta de medicamentos para a realização deste. Ainda assim, constatou-se que a maior parte das mulheres entrevistadas não haviam perdido a esperança de serem chamadas pelo serviço para engravidar. A preocupação com a eqüidade admite que, ao aguardar por tempo indeterminado na fila para obter tratamento de fertilização, essas mulheres têm seu direito de saúde afetado e passam a fazer parte dos grupos vulneráveis que tendem a suportar proporções indevidas de problemas de saúde. Portanto, o portador dos direitos freqüentemente transita nos frágeis laços entre o princípio da justiça e o funcionamento das instituiç̧ões e seus sistemas de garantia de acesso à saúde pública.

Tendo em vista os resultados da pesquisa de campo, é possível apontar, à luz da Bioética, que o Estado precisa rever a forma como o tratamento de RA está sendo oferecido por meio do SUS, pois não corresponde às expectativas das mulheres à espera desse tratamento. Cumpre lembrar que, em um Estado onde há liberdade de escolha mas os recursos são escassos para atender às necessidades e expectativas da população, uma atenção adequada à saúde deve levar em conta as dificuldades de acesso aos bens oferecidos no planejamento da alocação de recursos. Sendo assim, se o Estado disponibiliza ao cidadão o acesso à RA, mas esta tecnologia está longe de seu alcance; pela escassez da oferta, pode-se dizer que o princípio da eqüidade na saúde pública brasileira, neste caso, não está atingindo aqueles que precisam ser vistos na sua individualidade.

A responsabilidade do Estado em fomentar a expectativa dessas mulheres de procriar estabelece uma relação de descaso quanto aos vulneráveis, pois, a esperança do casal existe e persiste; porém, o serviço oferecido não é capaz de suprir o desejo ou necessidade de engravidar, tornando os casais pobres ainda mais distanciados de benefícios supostamente garantidos constitucionalmente.

\section{Agradecimento}

Os autores agradecem o apoio da Dra. Magaly Rulli Costa (HRAS-DF) no desenvolvimento da pesquisa.

\section{Conflito de interesse: não há.}

\section{SUMMARY}

Bioethical STUdY ON THE EXPECTATIONS OF WOMEN AWAITING assisted reproduction in a pUblic hospital in the Federal District, Brazil

OBJECTNE. Toanalyze the expectations of women who waitfor Assisted Reproduction Treatment-RA in the publichospital chosen as the reference in the Public Health Network in the Federal District - HRAS, Brazil.

Methods. For thirty days, 5 I women of the 56 who went to the Hras for infertility treatment were interviewed by a questionnaire including 10 objective questions related to the topic.

RESULTS. This trial was divided into two groups. The first, the "control group", comprised 27 patients recently sent to the reference public hospital from local health care centers or a regional hospital. The second, the "study group", comprising 24 women already diagnosed by the medical staff of HRAS and in the waiting line for "in vitro" fertilization. According to the input provided by the two groups, results show that the average waiting time for treatment is so long that women actually age during this time and face the risk of having a dangerous pregnancy before they receive treatment.

CONCLUSION. These results show that women unable to pay for treatment in a private fertilization clinic have a poorer chance of achieving RA: the health problem concerning this specific population ignores redressing or income distribution processes. Data show that, notwithstanding, this waiting period imposed by the State, expectations of the patients waiting for $R A$ are reinforced. There is $n$ basis to provide information about the waiting time. The unpredictable availability of the medication needed for in vitro fertilization, jeopardizes the future of this service offering the treatment. [Rev Assoc Med Bras 2007; 53(I): 47-52]

KEY WORDS: Bioethics. Unified Public Health System. Assisted reproduction. Equity. Priority. 


\section{REFERÊNCIAS}

I. Brasil, Constituição I988. Brasília: Senado Federal, Centro Gráfico; 1988.

2. Declaração Universal dos Direitos Humanos. Artigo I6. Assembléia Geral das Nações Unidas, 1948. Disponível em: http://www.mj.gov.br/sedh. [acesso em: 10 dez. 2004].

3. Costa R. Reprodução e gênero. Rev Est Fem. 1999; I 0(2):339.

4. Brasil. Diário Oficial da União (Brasília), Seção I, de 19-12-1992, p. 16.053.

5. Projetos de Lei (PL) n. 206 |/2003, I | 84/2003, I | 35/2003 e I 20/2003. Disponível em: http//www.camara.gov.br. [acesso em: 6 nov. 2004].

6. Dossiê Reprodução Assistida. Disponível em: http//:www.sbra.com.br. [acesso em: 6 nov 2004].

7. Dossiê Reprodução Humana Assistida. Rede Feminista de Saúde. Rede Nacional Feminista de Saúde, Direitos Sexuais e Direitos Reprodutivos, 2003. Disponível em: http//:www.redesaude.org.br/dossies. [acesso em: I 5 nov. 2004]

8. Ventura, M. Direitos reprodutivos no Brasil. São Paulo: Mventura; 2002.

9. Fundo de População das Nações Unidas - UNFPA. Dez anos do Cairo: tendências da fecundidade e direitos reprodutivos no Brasil. Brasília(DF); 2004.

10. Vanderplaat M. Núcleo de Estudos em Direitos Humanos e Saúde. In: Fundação Oswaldo Cruz. Saúde e direitos humanos. Brasília: Ministério da Saúde; 2004. p.30.

I I. Silva, S. Inovação nas políticas populacionais: o planejamento familiar no Brasil. Rev Eletrônica de Geografia e Ciência Sociais. 2000; 69.

12. Cunha ACR. Reflexão bioética na laqueadura tubária em mulheres com desejo de nova gestação [tese]. Brasília: Universidade Federal de Brasília; 2005.
13. "SUS tem recorde de laqueaduras e vasectomias". O Estado de São Paulo, São Paulo, 2004 julho 7. p.A9

14. "Governo quer ampliar ações de planejamento familiar na rede pública de saúde". Radiobrás. Disponível em: http//www.radiobras.gov.br. [Acesso em: 8 fev. 2005].

15. "Maternidade adiada". Correio Braziliense. Brasília (DF) 2002 julho 25. p.26.

16. Garrafa V. Dimensão da ética em saúde pública. São Paulo: Faculdade de Saúde Pública/USP Kellogg Foundation; 1995.

17. Rawls J. Uma teoria da justiça. São Paulo: Martins Fontes; 1997.

18. Lacoste J. A filosofia no Século XX. Campinas: Papirus; 1992.

19. Sen A. desigualdade reexaminada. Rio de Janeiro: Record; 2001.

20. Garrafa V, Oselka G, Diniz D. Saúde pública, bioética e eqüidade. Bioética (CFM). 1997;5(I):27-33.

21. Berlinguer G. Bioética cotidiana. Brasília: Editora UnB; 2004.

22. Kottow M. Bioética e política de recursos em saúde. In: Garrafa $\vee$, Costa IF, organizadores. A bioética no Século XXI. Brasília: Editora UnB; 2000.

23. Ministério da Saúde. Relatório do Conselho Nacional de Saúde: O desenvolvimento do Sistema Único de Saúde. Brasília: Editora MS; 2002.

Artigo recebido: 02/01/06

Aceito para publicação: 01// I/06 